\title{
Impact of IVC Filter Guidelines, Registry, and Clinic on Filter Retrieval.
}

\author{
Brandon Kiley ${ }^{1}$, BS, Lisa Hollister ${ }^{2}$, MSN, RN, T. Eric White ${ }^{3}$, MD, Emily Keltner ${ }^{3}$, BS, MA, Thein
} Zhu $^{2}$, MBBS, FACE, FRCP, Dazar Opoku², MPH

${ }^{1}$ Indiana University School of Medicine, ${ }^{2}$ Parkview Trauma Services, ${ }^{3}$ Parkview Heart Institute

Background and hypothesis: Deep vein thrombosis (DVT) and pulmonary embolism (PE), collectively referred to as venous thromboembolism (VTE), are serious medical conditions that affect up to 900,000 Americans yearly, accounting for up to 100,000 deaths. The first line treatment for VTE is anticoagulation; however, in patients who experience a contraindication to, or failure of anticoagulation, an IVCF may be used. There are two types of IVCFs, permanent and retrievable. Retrievable filters are indicated when the contraindication to anticoagulation is transient, and they may be removed once the contraindication has passed. Retrievable filters have become associated with serious complications such as filter fracture, migration, and IVC perforation. Subsequently, they have become the subject of litigation. As such, strategies should be undertaken to reduce filter dwell time and improve filter retrieval rates. We hypothesize that implementation of IVCF guidelines, registry, and clinic will reduce dwell time while increasing retrieval rate.

Methods: This study was a mixed retrospective and prospective chart review of patients who received an IVCF before and after implementation of IVCF guidelines, registry, and clinic. The guidelines, registry, and clinic were established in July 2017. Cases were analyzed during the years 2014-2015 ( $n=191)$ and 2017-2018 ( $n=103)$ beginning in July 2017. Data was obtained on filter retrieval rate, dwell time, filter-associated complications, and indication for placement.

Results: There was a significant decrease in dwell time $(p<.001)$ and a significant increase in retrieval rate $(p<.001)$. There was no difference in complication rate, and there was a decrease in filter placement in patients with 'soft' indications, though this difference was not statistically significant $(p=.109)$.

Conclusion and potential impact: Implementation of dedicated efforts to increase patient follow-up and filter retrieval were effective in reducing dwell time and retrieval rate. Although there was no significant difference in complication rate, these efforts may be protective against litigation for patients who experience a filter-associated complication. 